\title{
Monitoring in real-time focal adhesion protein dynamics in response to a discrete mechanical stimulus
}

Catalina von Bilderling, Martín Caldarola, Martín E. Masip, Andrea V. Bragas, and Lía I. Pietrasanta

Citation: Rev. Sci. Instrum. 88, 013703 (2017); doi: 10.1063/1.4973664

View online: http://dx.doi.org/10.1063/1.4973664

View Table of Contents: http://aip.scitation.org/toc/rsi/88/1

Published by the American Institute of Physics

\section{Articles you may be interested in}

A simple photoacoustic detector for highly corrosive gases

Rev. Sci. Instrum. 88, 013103013103 (2017); 10.1063/1.4972584

Large field-of-view tiled grating structures for X-ray phase-contrast imaging

Rev. Sci. Instrum. 88, 015104015104 (2017); 10.1063/1.4973632

Rotatable Offner imaging system for ellipsometric measurement

Rev. Sci. Instrum. 88, 013704013704 (2017); 10.1063/1.4973778

An integrated device with high performance multi-function generators and time-to-digital convertors

Rev. Sci. Instrum. 88, 014702014702 (2017); 10.1063/1.4973725

\section{SHIMADZU Powerful, Multi-functional UV-Vis-NIR and} Excellence in Science FJus Spectsophotometers

Providing the utmost in sensitivity, accuracy and resolution for applications in materials characterization and science

- Photovoltaics

- Polymers

- Coatings

- Ceramics

- Thin films

- Inks

- DNA film structures

- Packaging materials

- Nanotechnology

Click here for accurate, cost-effective laboratory solutions

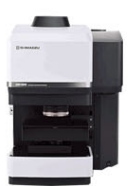

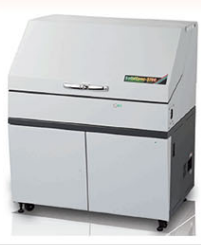




\title{
Monitoring in real-time focal adhesion protein dynamics in response to a discrete mechanical stimulus
}

\author{
Catalina von Bilderling, ${ }^{1,2}$ Martín Caldarola, ${ }^{3, a)}$ Martín E. Masip,, $\left.{ }^{3, b}\right)$ Andrea V. Bragas, ${ }^{2,3,4, c)}$ \\ and Lía I. Pietrasanta ${ }^{1,2,4, c)}$ \\ ${ }^{1}$ Centro de Microscopías Avanzadas and Departamento de Física, Facultad de Ciencias Exactas y Naturales, \\ Universidad de Buenos Aires, Buenos Aires, Argentina \\ ${ }^{2}$ IFIBA-CONICET-UBA, Buenos Aires, Argentina \\ ${ }^{3}$ Laboratorio de Electrónica Cuántica, Departamento de Física, Facultad de Ciencias Exactas y Naturales, \\ Universidad de Buenos Aires, Buenos Aires, Argentina \\ ${ }^{4}$ Consejo Nacional de Investigaciones Científicas y Técnicas (CONICET), Buenos Aires, Argentina
}

(Received 24 May 2016; accepted 22 December 2016; published online 11 January 2017)

\begin{abstract}
The adhesion of cells to the extracellular matrix is a hierarchical, force-dependent, multistage process that evolves at several temporal scales. An understanding of this complex process requires a precise measurement of forces and its correlation with protein responses in living cells. We present a method to quantitatively assess live cell responses to a local and specific mechanical stimulus. Our approach combines atomic force microscopy with fluorescence imaging. Using this approach, we evaluated the recruitment of adhesion proteins such as vinculin, focal adhesion kinase, paxillin, and zyxin triggered by applying forces in the $\mathrm{nN}$ regime to live cells. We observed in real time the development of nascent adhesion sites, evident from the accumulation of early adhesion proteins at the position where the force was applied. We show that the method can be used to quantify the recruitment characteristic times for adhesion proteins in the formation of focal complexes. We also found a spatial remodeling of the mature focal adhesion protein zyxin as a function of the applied force. Our approach allows the study of a variety of complex biological processes involved in cellular mechanotransduction. Published by AIP Publishing. [http://dx.doi.org/10.1063/1.4973664]
\end{abstract}

\section{INTRODUCTION}

Mechanical forces have been demonstrated to be essential in the function, organization, growth, and maturation of living tissues. ${ }^{1}$ Cells constantly sense and respond to their physical microenvironment through a process known as mechanotransduction, by which a mechanical stimulus can be converted into a biochemical response. These specific mechanosensing mechanisms, mediated by the actin cytoskeleton, can be modulated by a wide range of forces. ${ }^{2,3}$ Focal adhesions (FAs), the specialized multiprotein structures that mediate mechanotransduction at the cell-matrix level, are dynamic complexes that can undergo assembly, disassembly, and movement. Their composition involves more than 200 proteins, ${ }^{4,5}$ from which the extracellular matrix (ECM) protein fibronectin is known to trigger a force-driven assembly of focal complexes. ECMcytoskeleton linkage is also mediated by anchor cytosolic proteins, organized into a composite multilaminar architecture, ${ }^{6}$ some of which mediate the actin-membrane connection, whereas others have a regulatory function. ${ }^{4,7}$

The study of mechanotransduction responses requires the correlated measurement of mechanical stimuli and real time live-cell responses. Several approaches have been designed to

\footnotetext{
a) Current address: Leiden Institute of Physics, Leiden University, The Netherlands.

b) Current address: Max Planck Institute of Molecular Physiology, Dortmund, Germany.

c) Authors to whom correspondence should be addressed. Electronic addresses: bragas@df.uba.ar and lia@df.uba.ar.
}

assess this challenge, using external mechanical stimuli that range from forces globally applied to the cell (such as fluid shear stress ${ }^{8}$ or using stretching devices ${ }^{9,10}$ ) to specific and locally applied forces, generally involving the use of fibronectin (or fibronectin-fragments)-modified beads and tweezers to exert/sense the forces. ${ }^{11,12}$ Reported responses to these mechanical stimuli include the protein accumulation ${ }^{11}$ and translocation, ${ }^{13}$ and the activation of small GTPases of the Rho family. ${ }^{9}$

Atomic force microscopy (AFM) has become a powerful tool for nanobiotechnological investigations, capable to exert and probe forces on single cells ${ }^{14}$ and molecules. ${ }^{15,16}$ The combination of AFM and fluorescence microscopy has been applied successfully to various systems where the response to a precise mechanical stimulus must be monitored. Examples include the study of changes of intracellular calcium concentration, ${ }^{17}$ the formation of an actin perinuclear ring, ${ }^{18}$ and the rearrangement of vinculin and actin upon Rho-Ainduced cytoskeletal tension. ${ }^{19} \mathrm{AFM}$ was also combined with side-view optical imaging ${ }^{20}$ to study the response in terms of cell shape and actin cytoskeleton to a contraction of the whole cell between the AFM probe cantilever and a glass substrate. However, these studies generally analyze global cell responses such as cell shape or total protein area, and very few of them quantify the temporal evolution of these responses with spatial resolution. In this work, we describe the integration of an AFM with an epifluorescence microscope ${ }^{21}$ to investigate the dynamics of adhesion proteins within single FA in response to a controlled local mechanical stimulus. Indeed, AFM combines the possibility of applying forces from $\mathrm{pN}$ to 
$\mathrm{nN}$, localized in a contact area that can be down to a few $\mathrm{nm}$ for typical AFM-probes. A mechanical load was exerted on a living cell surface by a fibronectin-modified AFM probe as time-dependent spatial redistribution of the adhesion proteins vinculin, focal adhesion kinase (FAK), paxillin, and zyxin was monitored in time. With this methodology, we are able to observe the development of a nascent focal site triggered by a local mechanical stimulus. A quantitative analysis of the fluorescence intensity allowed us to evaluate characteristic protein recruitment times in the force induced assembly of focal complexes. In addition, we observed a spatial distribution remodeling of the protein zyxin in mature FAs as a function of an increasing applied force.

\section{EXPERIMENTAL METHODS}

\section{A. Mechano-response experiment}

The formation of adhesion sites requires the specific interaction between fibronectin and integrin receptors, ${ }^{11,19}$ as well as the action of an external $\mu \mathrm{m}$-localized mechanical stimulus. ${ }^{11}$ Therefore, in order to stimulate the formation of new adhesion sites, we use a fibronectin-modified AFM probe to exert localized and controlled $\mathrm{nN}$ forces on the cells, and to evaluate how this combined functional mechanical stimulus triggers the recruitment of proteins during focal complexes formation (Fig. 1(a)).

Wide-field fluorescence microscopy, broadly used to study FA, ${ }^{11,22-27}$ is an excellent tool for recording the protein dynamics in real time while applying the mechanical stimulus at the apical cell surface. Cell thickness at the zone, where the localized stimulus was made, is of at least of $2 \mu \mathrm{m}$ (evaluated directly from AFM topography images), while cell indentation with AFM tips was always about $500 \mathrm{~nm}$ (calculated from the exerted force values and cantilever spring constants, Fig. 1(b)). Therefore, the need of simultaneous imaging of mature FA at the basal cell level as well as nascent FX at the AFM tip makes the wide-field fluorescence microscopy the best optical technique for carrying out these experiments.

The enhanced green fluorescent fusion proteins (EGFP)vinculin, EGFP-FAK, EGFP-paxillin, or EGFP-zyxin were expressed in mouse mammary epithelial cells (HC11 cell line, Figure 1 of the supplementary material). Time-lapse live-cell imaging was performed at room temperature in RPMI medium without Phenol Red (Invitrogen) and supplemented with $25 \mathrm{mM}$ Hepes buffer (Sigma). Cell viability at these conditions was verified through a Propidium Iodide viability assay (data not shown).

For our experimental mechanotransduction assay (Fig. 1(a)), only cells expressing the EGFP-FA fusion proteins are selected under the fluorescence microscope and a region close to the periphery of the cell is chosen to place the AFM probe on top. The AFM contact mode approach curve is performed under a low force setpoint (typically $<10 \mathrm{nN}$ ) to ensure that the AFM probe is on the cell surface having an indentation shape. Then, the focus is corrected if necessary, and the setpoint is changed to reach the desired force value (generally of tens of $\mathrm{nN}$ ). This moment is defined as $\mathrm{t}=0$ (time zero) of the experiment, and the recording of the fluorescence images starts. The CCD camera integration time is defined previously depending on the fluorescence intensity in each selected cell (from 1 to $5 \mathrm{~s}$ ). For experiments performed in a cell at different force levels, the time $t=0$ for each experiment (performed sequentially from the lowest force value to the highest one) is defined as the moment at which the setpoint is changed from one force value to the following one.

AFM sensitivity, i.e., the ratio between the AFM detector signal and the $\mathrm{z}$ displacement of the probe in contact with a rigid surface, was calibrated for each experiment and in the same conditions, by taking force curves under fluid in a region free of cells of the same coverslip (to guarantee a rigid surface and the absence of indentation).

\section{B. Combined AFM and fluorescence microscopy}

For the combined AFM-optical microscope (Fig. 2(a)), the control electronics (RHK Technology, SPM100-AFM100) acquires and processes the four-quadrant detector signal generated in a home-made AFM head, which accounts for the cantilever deflection. The electronics also manage the feedback loop as well as the axial and lateral movements of the sample, through a XYZ piezoelectric-stage (Physik Instrumente (PI),
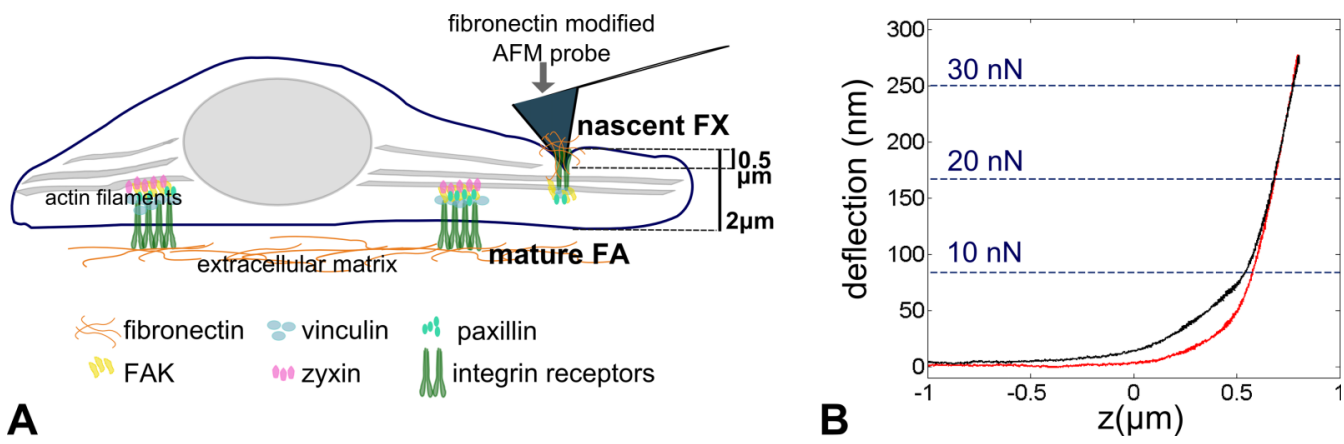

FIG. 1. (a) Schematic representation of our experimental mechanotransduction assay. Fibronectin-modified AFM probe applies a mechanical stimulus pushing down at the apical surface of living cells. As a result of this functional mechanical stimulus, transient FA protein responses are expected: the formation of a new adhesion sites as well as the remodeling of mature FA. Note that zyxin is a component only of mature focal adhesions while vinculin, FAK, and paxillin are present throughout the lifetime of focal adhesions. Typical AFM probes indentation distances and cell thickness (at the point where the force was applied) are shown. (b) Approach (black) and retract (red) typical AFM indentation force curve at the cell surface. Deflection values corresponding to applied forces of 10, 20 , and $30 \mathrm{nN}$ are marked with horizontal lines. Applied force stimuli (in the order of tens of $\mathrm{nN}$ ) correspond to indentation distances of approximately $0.5 \mu \mathrm{m}$ (always below $1 \mu \mathrm{m}$ ) as is depicted in (a). 

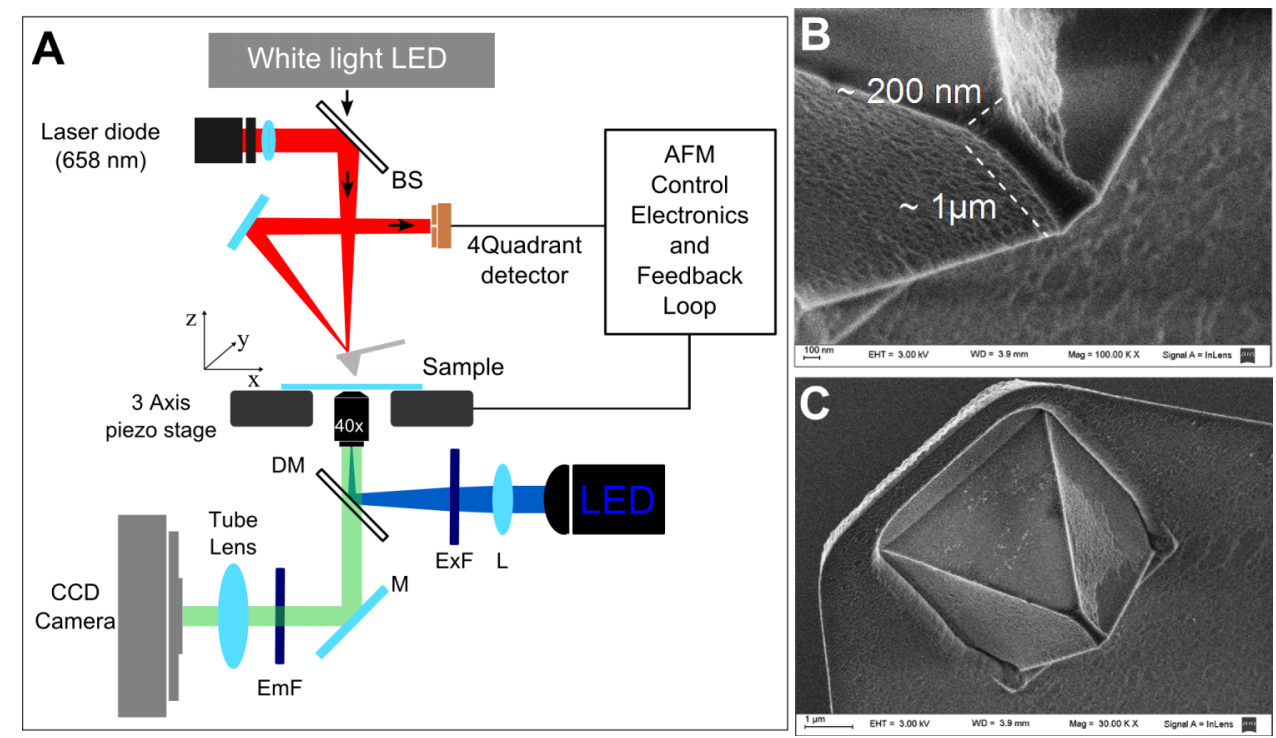

FIG. 2. (a) Combined microscope setup: the control electronics acquire and process the home-made AFM detector signal (which accounts for the cantilever deflection), the feedback loop, and the axial and lateral movements of the sample (through a XYZ piezoelectric-stage with sub nanometer axial accuracy). The AFM works on top of an inverted wide-field fluorescence microscope (blue LED light source, CCD camera, and conventional filters). ((b) and (c)) Scanning electron microscopy image of a worn down AFM probe used in the experiments. The minimum contact area was increased to approximately $0.2 \mu \mathrm{m}^{2}$ (b), whereas the worn down pyramidal tip has a side length of above $3 \mu \mathrm{m}$ (c).

P517) with sub-nanometer axial accuracy. The AFM was operated in the contact mode under a liquid environment. AFM force spectroscopic capabilities in terms of force detection sensitivity were measured to reach single ligand-receptor interaction forces in a model system as biotin-streptavidin. ${ }^{21}$

The AFM head works on top of an inverted multipurpose optical microscope, which was operated as a wide field fluorescence microscope, with a blue LED (Tolket, SRL) as the light source, conventional GFP filter set (Chroma), and a CCD camera (Apogee Imaging Systems, Alta U2000). Two notch filters block the red AFM laser from the optical microscope light path. Image acquisition was performed with custommade LabView software. Time-lapse imaging allows following the FAs without losing the microscope focus during the time lapse of the experiments.

Background level of the fluorescence images was different under the AFM cantilever position. This "shadow" of the AFM cantilever, evidenced or not at the images depending on the selected exposure time (between 0.5 and $5 \mathrm{~s}$ ), was properly characterized for each experiment and taken into account for background corrections (see Sec. II E).

\section{AFM probe wearing and functionalization}

Silicon nitride triangular cantilevers (NP-STT) from Veeco Instruments (Santa Barbara, CA, USA, actually Bruker) were used. The softest cantilevers were chosen, which have $205 \mu \mathrm{m}$ length and $25 \mu \mathrm{m}$ width (for the cantilever with nominal spring constants of $0.06 \mathrm{~N} / \mathrm{m}$ ) or $40 \mu \mathrm{m}$ width (nominal spring constant of $0.12 \mathrm{~N} / \mathrm{m}$ ).

Ligand surface area has been demonstrated to be determinant on the ability to form focal complexes. ${ }^{11}$ Formation of adhesion sites has been generally performed using fibronectin functionalized beads with diameters of more than $1 \mu \mathrm{m}$. Typical AFM tip radii are of a few nanometers, leading to a minimum ligand contact area. Therefore, in order to promote the formation of focal complexes with our methodology, we worn down the AFM tips to reach a minimum contact surface in the same order of magnitude of the used on published works. ${ }^{11,19}$ Prior to the molecular functionalization, the AFM probes were worn down to significantly increase the contact area to approximately $0.2 \mu \mathrm{m}^{2}$ (Figs. 2(b) and 2(c)). This process was performed using a commercial AFM (Multimode AFM, Bruker, Santa Barbara, CA) by scanning in contact mode an $1 \mu \mathrm{m} \times 1 \mu \mathrm{m}$ area at high velocity (60 lines/second) and maximum setpoint in MilliQ water. AFM probes were also coated with gold $(\sim 10 \mathrm{~nm}$ thickness) in a sputter coater $(108$ Sputter Coater, Kurt J. Lesker Co.) to facilitate the adsorption of fibronectin molecules. Tips were washed with ethanol and dried under nitrogen after the wearing down and sputtering steps. Finally, the force probes were incubated in a $100 \mathrm{mM}$ fibronectin (Sigma) solution (in phosphate buffered saline, PBS) for $1 \mathrm{~h}$ at room temperature (RT), washed gently five times with PBS to remove non-attached proteins, and maintained in PBS until used.

The cantilever spring constants for each force probe were determined in air using the thermal tune method, ${ }^{28}$ after the wearing down and sputtering steps and before fibronectin incubation. Calibrated spring constants range from $0.035 \pm 0.003$ to $0.12 \pm 0.01 \mathrm{~N} / \mathrm{m}$. The spring constant of the cantilever was assumed to be unchanged after the protein functionalization step.

\section{Cell culture and transfection}

HC11 mammary epithelial cells ${ }^{29}$ (a kind gift from Dr. Nancy Hynes) were cultured in supplemented RPMI1640 media (Invitrogen) with 10\% FBS (Internegocios S.A.), $100 \mathrm{IU} / \mathrm{ml}$ penicillin $100 \mu \mathrm{g} / \mathrm{ml}$ streptomycin (Invitrogen), and $5 \mu \mathrm{g} / \mathrm{ml}$ insulin from bovine plasma (Sigma-Aldrich) and 
incubated at $37^{\circ} \mathrm{C}, 5 \% \mathrm{CO}_{2}$. The cells were grown in a T25 tissue culture flask until they reached the appropriate density. Then, the cells were rinsed with PBS and incubated with $1 \mathrm{ml}$ trypsin/EDTA (Invitrogen) at $37^{\circ} \mathrm{C}$ for $5 \mathrm{~min}$ until the cells detached. To inactivate the trypsin, $4 \mathrm{ml}$ of growth medium was added and the cells were diluted according to their growth rate.

Coverslips for cell culture (24-mm diameter) were incubated with a PBS solution of $1 \mathrm{mg} / \mathrm{ml}$ fibronectin (SigmaAldrich) for $30 \mathrm{~min}$. The solution was removed, and the coverslips were dried and kept in a dust-free container until use. The day before transfection, $3-5 \times 10^{5}$ cells were seeded on fibronectin-coated coverslips and maintained in growth media, at $37^{\circ} \mathrm{C}$ with $5 \% \mathrm{CO}_{2}$. The DNA constructs EGFPzyxin, EGFP-vinculin, EGFP-paxillin, and EGFP-FAK were kind gifts from groups of Ingber, Geiger, Tamada, and Parsons, respectively. Cells were transfected on 6-well plates using Lipofectamine 2000 (Invitrogen). Briefly, $4 \mu \mathrm{l}$ of Lipofectamine and separately $2 \mu \mathrm{g}$ of plasmid-DNA were diluted in $50 \mu \mathrm{l}$ OptiMEM (Invitrogen) and incubated at RT for $5 \mathrm{~min}$. Both solutions were mixed and incubated for $30 \mathrm{~min}$ at RT. Lipofectamine-DNA complexes were added to the cells in a drop-wise manner, and the chambers were subsequently swirled to ensure a homogenous distribution of the complex over the entire surface. The cells were incubated at $37^{\circ} \mathrm{C}$ with $5 \% \mathrm{CO}_{2}$ for $2 \mathrm{~h}$, rinsed with PBS, and maintained in growth medium until observation. Experiments were performed between 24 and $48 \mathrm{~h}$ after transfection to ensure protein expression.

\section{E. Data collection and analysis}

Image sequences were acquired with the combined microscope using a $40 \times 0.75 \mathrm{NA}$ objective lens (Carl Zeiss) at $1200 \times 1600$ pixels (binning $1 x$ ). When required, the images from the sequences were aligned to correct experimental drift using the FIJI plugin "align slices in stack." All image processing and analysis were performed with custom-written Matlab routines.

To assess for intensity changes at the images sequence, we defined the ratio after/before (Ratio after/before $_{\text {) }}$ as the intensity ratio between the mean of the last 20 images (I(end)) and the mean of the first 20 images $(I(0)$, both background subtracted and bleaching corrected) of the sequence minus one, taken pixel by pixel as follows:

$$
\text { Ratio }_{\text {after } / \text { before }}=\frac{I(\text { end })}{I(0)}-1 .
$$

This ratio accounts for changes in fluorescence intensity when the last and first images are compared, taking positive values when fluorescence increases, negative values when fluorescence decreases, and values near zero in pixels of the image without changes in fluorescence. An analog procedure was followed for intensity evolution analysis: Regions of interest (ROIs) within FA were selected, for which the fluorescence intensity $(I(t))$ was quantified as the mean intensities in the ROI (i.e., mean intensity per pixel in the region), as a function of the time. The intensities were calculated and the normalized intensity used for the analysis was calculated from the following:

$$
I_{\text {normalized }}(t)=\frac{I(t)}{I(0)}-1 .
$$

Intensities used in all calculations were background subtracted and bleaching corrected as follows:

- Background (bkg) was quantified in regions without cells at the image, both in the region under the cantilever $\left(b k g_{\text {cant }}\right)$ and outside from the cantilever shadow $\left(b k g_{\text {out }}\right)$. Raw intensities at each pixel were then background corrected by subtracting $(I=I-b k g)$, where $b k g$ is either $b k g_{\text {out }}$ or $b k g_{\text {cant }}$ depending on the location of the pixel.

- Photobleaching over time $(P B(t))$ was evaluated by averaging background corrected intensities from pixels inside regions corresponding to control FA. Control FAs were selected from the mature FA at cells not stimulated by the AFM tip or at the stimulated cell but far away from the tip. All control FAs presented the same dynamic behavior: an exponential decay of the same characteristic time coming from the image acquisition photobleaching.

When characterizing the characteristic recruiting times, we performed a fit to recover the temporal constant $(\tau)$ of an exponential function according to the following:

$$
I(t)=A\left(1-e^{-t / \tau}\right)+B .
$$

\section{EXPERIMENTAL RESULTS}

\section{A. Localized protein recruitment in response to the functional mechanical stimulus}

Temporal evolution of the fluorescence intensity of (EGFP)-vinculin was examined while applying a force of $66 \pm 6 \mathrm{nN}$ (Fig. 3) with a fibronectin functionalized AFM probe and followed frame by frame for $250 \mathrm{~s}$. Here, we define as time zero the instant in which the selected force value (setpoint) is applied. However, the interaction may be started a few tens of seconds before the AFM probe was gently moved toward the cell to start the experiment. A live-cell AFM image (Fig. 2(b)) once finishing the experiment confirmed the cell membrane preservation after the force stimulus was applied.

In Figs. 3(c) and 3(d) we show images of the region covered by the AFM probe at different experiment times, in which spatial distribution of changes in vinculin intensity is evidenced. The ratio after/before map, as defined in Sec. II (Sec. II E), accounts for changes in fluorescence intensity when the last and first images are compared. The pseudocolor scale assigns red pixels to positive ratio values (fluorescence increases), blue pixels to negative ratio values (fluorescence decreases), and gray pixels to regions without changes (fluorescence does not change). This map (Fig. 3(e)) allows us to quantify the remarkable changes in fluorescence signal intensity over the time. The arrows point to the most dynamic regions of vinculin: a diffuse aggregate that evolved to a more condensed form following the location and shape of the fibronectinmodified AFM probe, as well a small nascent adhesion site at the vicinity of the tip. Time evolution of the normalized 


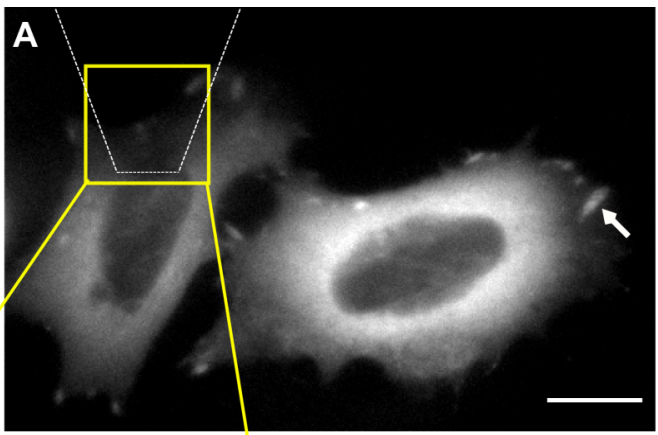

B
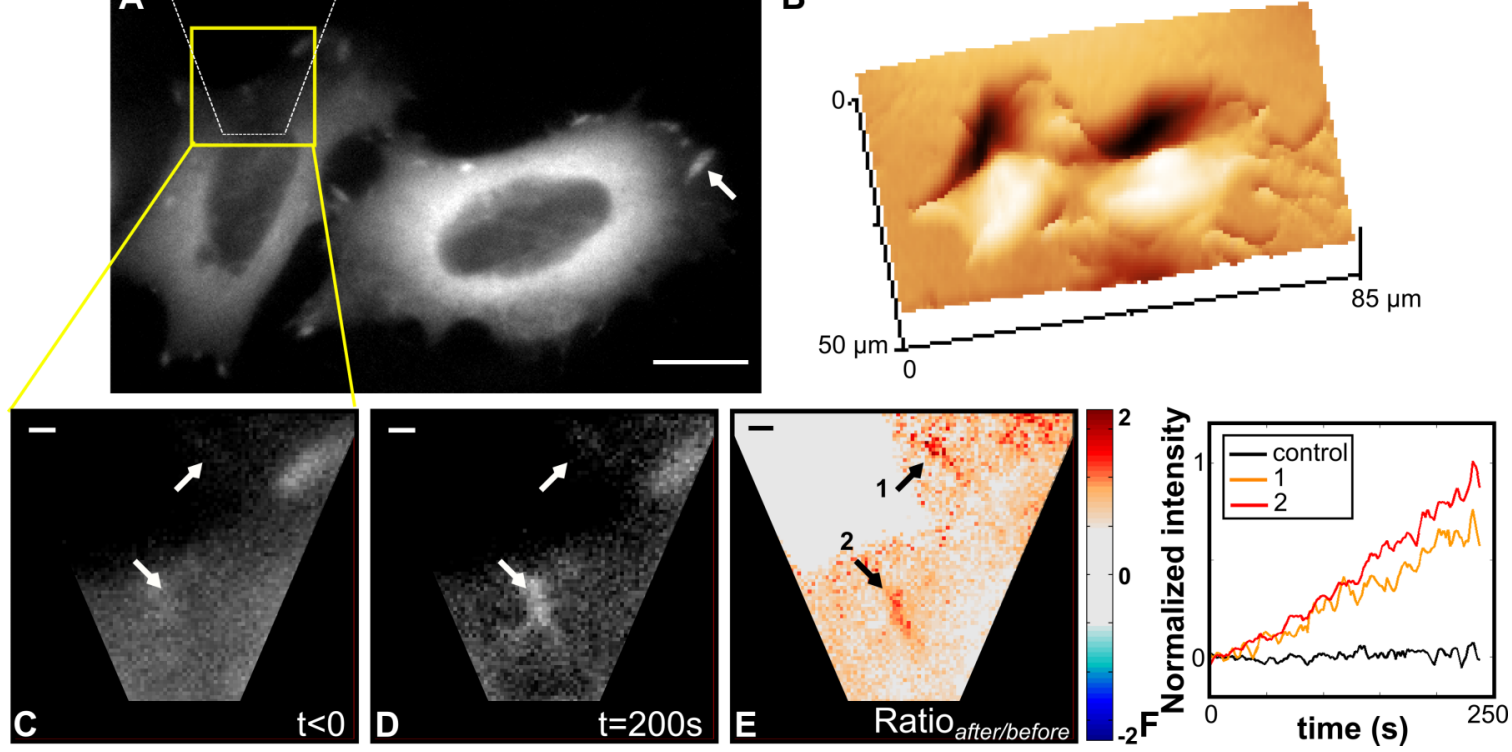

FIG. 3. (a) Fluorescence image of HC11 cells expressing EGFP-vinculin. Dashed white lines indicate the region under the AFM probe. Scale bar $10 \mu$ m. (b) AFM image of the same cells after finishing the time-lapse fluorescence experiment. ((c) and (d)) Selected frames at indicated times (relative to the setpoint change) reveal the changes in the fluorescence intensity over the time. Vinculin is clearly recruited at the AFM probe apex as well as to a region on the upper right corner at a slower rate. (e) The ratio after/before of the fluorescence intensities shows an above twofold increase of the intensity in some pixels (ratio $>1$ ), which is also evidenced by quantifying the temporal evolution of the fluorescence intensity (f) at the two pointed regions (quantifying area was selected using an intensity threshold at the final image), as well as at a control region corresponding to a mature FA in a non-stimulated cell (pointed in (a)).

fluorescence intensity was quantified on regions coincident with the final shape of the protein aggregates (see Fig. 3(f)). An almost twofold increase of the mean intensity (final normalized intensity $=1$ ) demonstrates that the external local force applied from a functionalized AFM probe triggers vinculin recruitment and the development of nascent adhesion sites.

The formation of focal complexes was observed through the recruitment of vinculin, FAK, and paxillin. Accumulation of proteins typically displayed one of the following patterns (Figure 1 of the supplementary material): (1) a diffuse accumulation of protein formed around the tip apex (Figure $2 \mathrm{~A}-\mathrm{B}$ and $\mathrm{F}$ of the supplementary material) or (2) a group of focal complexes assembled at the tip surroundings (Figure $1 \mathrm{C}-\mathrm{D}$ and $\mathrm{E}$ of the supplementary material). This is consistent with previous observations for mechanical functional stimulation through functionalized $\mu \mathrm{m}$-sized beads. ${ }^{11}$ Protein recruitment was measured in at least two cells from different cultures for each one of the three mentioned proteins.

\section{B. Direct quantification of characteristic protein recruitment times}

Although protein composition of early focal complexes and mature focal adhesions has been extensively identified, ${ }^{4-6}$ the complete picture of the assembly sequence of events has yet to be revealed. Formation and maturation of focal complexes involve complex molecular connections. FAK, for example, was postulated to be recruited to integrin-talin clusters, but an alternative model was proposed to recruit talin to new sites of $\beta 1$ integrin-mediated adhesion. ${ }^{30}$ On the other hand, a recent work evidenced that cytosolic adhesion proteins pre-assemble into building blocks before recruiting to adhesion sites. ${ }^{31}$ In this context, characteristic recruitment times in the assembly of adhesion proteins are key clues, as their changes could be associated with different binding pathways. Here we extend our method to quantify characteristic recruiting times of FAK and paxillin in the assembly of focal complexes triggered by AFM functional mechanical stimuli.

To determine the recruitment time for focal adhesion kinase (FAK), we performed time-lapse fluorescence microscopy of HC11 cells expressing EGFP-FAK. A functional local constant stress force of $37 \pm 9 \mathrm{nN}$ was applied for $1200 \mathrm{~s}$ to HC11 cells, and a wide-field fluorescence image was simultaneously recorded. Representative movie frames are provided in Figs. 4(b) and 4(c), where a selected region in the vicinity of the AFM probe apex is also shown. The ratio after/before map (Fig. 4(d)) reveals changes in the intensity of the fluorescence as well as in the spatial distribution at several zones of the images. Zone 1 (labeled with an arrow) is a region where the spatial concentration of the protein is occurring. During the first $900 \mathrm{~s}$, there were intensity changes, plotted in Fig. 4(e), from where a characteristic time of $(200 \pm 80) \mathrm{s}$ is recovered by fitting the data with an exponential function. This analysis was conducted on a ROI coincident with the final size of the FA as appears in Fig. 4(e). In addition, when looking at region 2, we observed the accumulation of the protein to a focal point with slower dynamics, perhaps because the recruitment already reached the plateau at the time of the experiment. Furthermore, there are other zones for which the protein recruitment starts at approximately $800 \mathrm{~s}$ (not shown), with no changes during the early stages of the experiment.

Similar results were observed for the recruitment of paxillin in response to a functional mechanical stimulus of $39 \pm 10 \mathrm{nN}$ (Figs. 4(f)-4(j)) for $1200 \mathrm{~s}$. Protein accumulation is observed as a group of focal complexes assembled at the tip surroundings. Among them, region 3 corresponds to a focal 

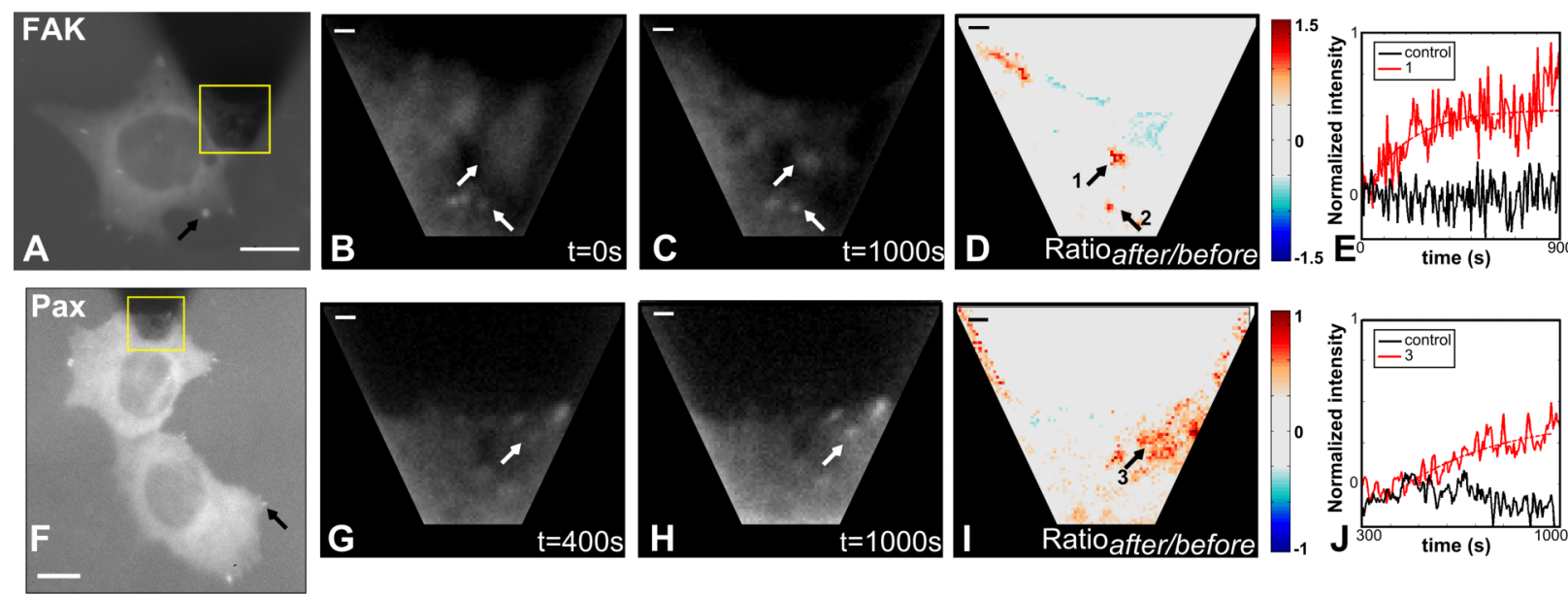

FIG. 4. (a) Fluorescence image of HC11 cells expressing EGFP-FAK. Square indicates the region for analysis, and arrow indicates a control FA. Scale bar $10 \mu \mathrm{m}$. ((b) and (c) Selected frames at indicated times (relative to the setpoint change) reveal the changes in the fluorescence intensity over the time. (d) The ratio after/before of the fluorescence intensities shows an increase of the intensity, which is also evidenced by quantifying the temporal evolution of the fluorescence intensity (e) at the pointed region (quantifying area was selected using an intensity threshold at the final image), as well as at a control region corresponding to a mature FA in a non-stimulated cell (pointed in A). Scale bars $2 \mu \mathrm{m}$. (f)-(j) Equivalent results for cells expressing EGFP-paxillin.

complex that begun to be recruited at approximately $400 \mathrm{~s}$ after the force was applied. The fit of the curve with an exponential increase function provided a characteristic recruitment time of $370 \pm 120 \mathrm{~s}$.

Vinculin as well as FAK has been identified as important mechano-regulatory players with similar mechanotransduction functions in the cell. ${ }^{32}$ Paxillin is another focal adhesionassociated signaling molecule that functions as an adaptor protein to recruit diverse cytoskeleton and signaling proteins. ${ }^{33}$ We found that vinculin, FAK, and paxillin are sensitive to an external mechanical perturbation of the same amplitude. In our experimental conditions, recruitment time of the FA protein vinculin (as can be estimated from temporal changes in the fluorescence intensity: slope of Fig. 3(f)), ranges in the same order of magnitude as recruitment times for FAK and paxillin, hundreds of seconds.

During the last decades, there has been a sustained interest on the study of focal complexes development. The hierarchical assembly picture is well established and the key component proteins are known, ${ }^{34-37}$ nevertheless the absolute timing of these events is still uncertain. Early FX proteins force-induced recruitment characteristic times from our results are in good agreement with the order of magnitude assessed in previous studies. ${ }^{11}$ Complete adhesion proteins recruitment time curves have been reported to our knowledge, only during initial cell assembly on planar supported lipid bilayer membranes with lipid-attached cyclic Arg-Gly-Asp (RGD) peptide ligands. ${ }^{38}$ Times of hundreds of seconds were also found on these conditions. The strategy presented here, feasible to perform at available commercial AFM-optical microscopes, represents an adaptable alternative to get new insights into the absolute timing of force induced FXs development.

\section{Mature FA proteins are recruited in response to discrete mechanical stimuli}

We also performed intensity evolution analysis in stable, mature focal adhesions (assembled at the cell-substrate interface before applying the force stimuli), in response to mechanical-functional stimulation. We found for vinculin, FAK, and paxillin that some of the mature focal adhesions, generally those positioned in the vicinity of the applied force location, have a different dynamic behavior as compared with control-cells focal adhesions (data not shown). These mature focal adhesion proteins dynamics were difficult to evaluate, as the predominant intensity changes are given by the assembly of new focal complexes. Therefore, to assess for changes in protein dynamics of mature focal adhesions, we use our method to perform studies in cells expressing the adhesion protein zyxin, which is a marker of mature focal adhesions and is notably absent from earlier focal complexes.

As expected at our experiment time scales, we did not observe zyxin recruitment at the apex of our functionalized AFM probe in response to the applied stimulus (zyxin is postulated to be recruited to FAs in tens of minutes). ${ }^{39}$ However, we observed changes in fluorescence intensity and spatial distribution of the protein in the mature (pre-assembled) FA located at the cell-substrate interface. These changes suggest a focal adhesion remodeling when an increasing force is applied to the apical cell surface. We are using the term remodeling to assess changes in the spatial distribution inside each FA.

Fig. 5(a) is the fluorescence image of a $\mathrm{HC} 11$ cell expressing EGFP-zyxin, in which the AFM probe location is plotted as a white line. We followed the time evolution of the fluorescence intensity in this cell for three different forces (setpoints) applied consecutively, $\mathrm{f}_{1}=50 \pm 8 \mathrm{nN}, \mathrm{f}_{2}=62 \pm 8 \mathrm{nN}$, and $\mathrm{f}_{3}=75$ $\pm 9 \mathrm{nN}$, during $250 \mathrm{~s}$ for each experiment. The yellow square indicates a zone, centered at the AFM probe apex (where the perturbation has been applied) for which a detailed analysis is presented in Figs. 5(b)-5(d). For this analysis, we plotted the ratio after/before maps, corresponding to the $f_{1}, f_{2}$ and $f_{3}$ experiments. The ratio after/before maps revealed spatial distribution changes in zyxin protein dynamics inside each one of the focal adhesions. The maximum changes in the ratio after/before are on a value near 0.4 , which is below the typical changes observed in nascent adhesions. It is striking that inside each 

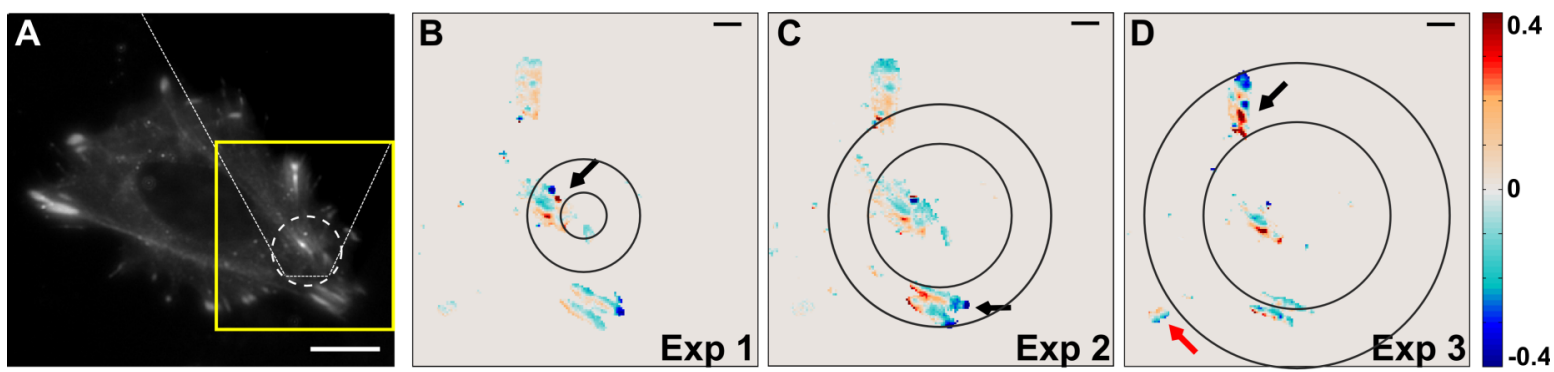

FIG. 5. (a) Fluorescence image of a HC11 cell expressing EGFP-zyxin. Scale bar $10 \mu \mathrm{m}$. Data were recorded just after applying a new setpoint force in three experiments performed at different force values: $\mathrm{f}_{1}=50 \pm 8 \mathrm{nN}(\operatorname{Exp} 1), \mathrm{f}_{2}=62 \pm 8 \mathrm{nN}(\operatorname{Exp} 2)$, and $\mathrm{f}_{3}=75 \pm 9 \mathrm{nN}$ (Exp 3). ((b)-(d)) Ratio after/before maps, at the region marked as a yellow square in (a), defined as the intensity ratio between the last and first images of the sequence for each one of the experiments at $\mathrm{f}_{1}$, $\mathrm{f}_{2}$, and $\mathrm{f}_{3}$, respectively. Inside each one of the FAs, there are zones where the protein is recruiting (red), whereas other zones display loss of protein (blue). These maps give direct evidence of the zyxin distribution remodeling in mature FA.

one of the FA, there are zones where the protein is recruiting (red), whereas other zones display loss of protein (blue), which confirms the force induced remodeling of zyxin within single mature FAs. This behavior was not evidenced while performing the same analysis for vinculin, FAK, and paxillin, for which only the protein recruitment at nascent adhesions dominated the fluorescence changes.

Moreover, when looking at the most dynamic regions for the successive experiments (whose boundaries were enclosed in circles centered at the force applied location in Figs. 5(b)-5(d), black arrows) it is evident that the remodeling propagates across regions located further away from the discrete stimulus, as the applied force is increased. Note that in Figure 5(d) FAs pointed with a red arrow, located far apart from the local applied force, presented a small dynamic behavior for the maximum applied force. These experiments showed that local forces applied at the apical cell surface (whose main component is vertical) are transmitted in a non-trivial manner to the cell-substrate plane where the mature focal adhesions are located.

\section{CONCLUSIONS}

It is widely recognized that mechanical forces are ubiquitous modulators in the organization, growth, maturation, and function of cells and organisms. Although significant progress has been achieved over the past decades in understanding cellular mechanotransduction, additional insights into the spatial and temporal sequence of events in cellular responses to mechanical stimuli are required to have one complete picture. ${ }^{40}$ In this study, we quantitatively assess the dynamics of adhesion proteins within single FAs in response to an applied local and functional mechanical stimulus. The present findings are the first experiments, to the best of our knowledge, in which the dynamics of proteins involved in the formation of FAs have been recorded in real-time after a discrete external functional mechanical stimulus has been applied. These experiments represent an important initial step in exploring the progression of the proteins recruitment into adhesion sites in response to an ECM-applied mechanical stimulus.

Our strategy combines AFM and fluorescence microscopy to quantitatively assess the dynamics of adhesion proteins within single FAs in response to an applied local and functional mechanical stimulus. Using this method, we demonstrated real-time (hundreds of seconds' length) measurements of vinculin, FAK, and paxillin recruitment to nascent FAs, with growth mostly around the fibronectin-modified AFM probe position, where the mechanical stimulus was applied. Quantitative analysis of the fluorescence intensity evolution allows the evaluation of an order of magnitude for the recruitment time of these proteins to FAs in response to an external stress, and in the cases of FAK and paxillin, a complete curve was recorded with characteristic times of $200 \pm 80 \mathrm{~s}$ and 370 \pm 120 s, respectively.

We also observed a spatial distribution remodeling of zyxin (accumulation-loss) within mature FA in response to applied force of different strengths. In addition, we found that the remodeling is spreading away from the applied stimulus as the force is increased. These results evidence a mechanical connection between the force contact point and mature focal adhesions.

Both nascent complexes and mature focal adhesions are distinct and functional roles for adhesion proteins within these structures may be different. The method presented in this work provides a new approach to answer questions as follows: how do cytosolic proteins get recruited to nascent focal complexes? Does the absence of one of these proteins alter the recruitment of the others? Are they recruited in pre-assembled blocks? What are the mechanisms of how the process of adhesion assembly and turnover are regulated by external applied forces? The activity of which of the proteins involved is forcesensitive? Focusing on either nascent or mature adhesions, when answering such questions the timing and spatial distribution are of key importance.

\section{SUPPLEMENTARY MATERIAL}

See supplementary material for confocal microscopy images of the adhesion protein expressing HC11 cells (Figure 1 of the supplementary material) and a gallery of images showing typical accumulation patterns of adhesion proteins around the AFM tip apex (Figure 2 of the supplementary material).

\section{ACKNOWLEDGMENTS}

This paper is dedicated to the memory of Dr. Elizabeth Jares-Erijman and in acknowledgment of her passion for life 
and work, inspiration, and unfailing support. We gratefully acknowledge Dr. Nancy Haynes for providing the cell line and Dr. D. E. Ingber, Dr. B. Geiger, Dr. K. M. Tamada, and J. T. Parsons for DNA constructs. We thank Dr. L. Sigaut and Dr. H. Grecco for helpful discussions and advice. We are indebted to Dr. F. Stefani for his encouragement and critical reading of the manuscript. C.v.B., M.C., and M.M. acknowledge CONICET for their doctoral fellowships. A.V.B. and L.I.P. are CONICET fellows.

${ }^{1}$ C. S. Chen, J. Cell Sci. 121, 3285 (2008).

${ }^{2}$ K. Burridge and C. Guilluy, Exp. Cell Res. 343, 14 (2016).

${ }^{3}$ A. Livne and B. Geiger, J. Cell Sci. 129, 1293 (2016).

${ }^{4}$ R. Zaidel-Bar, S. Itzkovitz, A. Ma' ayan, R. Iyengar, and B. Geiger, Nat. Cell Biol. 9, 858 (2007).

${ }^{5}$ E. Zamir and B. Geiger, J. Cell Sci. 114, 3583 (2001).

${ }^{6}$ P. Kanchanawong, G. Shtengel, A. M. Pasapera, E. B. Ramko, M. W. Davidson, H. F. Hess, and C. M. Waterman, Nature 468, 580 (2010).

${ }^{7}$ B. Geiger, A. Bershadsky, R. Pankov, and K. M. Yamada, Nat. Rev. Mol. Cell Biol. 2, 793 (2001).

${ }^{8}$ P. F. Davies, A. Robotewskyj, and M. L. Griem, J. Clin. Invest. 93, 2031 (1994).

${ }^{9}$ A. M. Goldyn, B. A. Rioja, J. P. Spatz, C. Ballestrem, and R. Kemkemer, J. Cell Sci. 122, 3644 (2009).

${ }^{10} \mathrm{M}$. Schwingel and M. Bastmeyer, PLoS One 8, e54850 (2013).

${ }^{11}$ C. G. Galbraith, K. M. Yamada, and M. P. Sheetz, J. Cell Biol. 159, 695 (2002).

${ }^{12}$ Y. C. Yung, J. Chae, M. J. Buehler, C. P. Hunter, and D. J. Mooney, Proc. Natl. Acad. Sci. U. S. A. 106, 15279 (2009).

${ }^{13}$ M. Yoshigi, L. M. Hoffman, C. C. Jensen, H. J. Yost, and M. C. Beckerle, J. Cell Biol. 171, 209 (2005).

${ }^{14}$ C. Rotsch and M. Radmacher, Biophys. J. 78, 520 (2000).

${ }^{15}$ P. Hinterdorfer and Y. F. Dufrene, Nat. Methods 3, 347 (2006).

${ }^{16}$ D. J. Muller, J. Helenius, D. Alsteens, and Y. F. Dufrene, Nat. Chem. Biol. 5, 383 (2009).

${ }^{17}$ G. T. Charras and M. A. Horton, Biophys. J. 82, 2970 (2002).

${ }^{18}$ X. Shao, Q. Li, A. Mogilner, A. D. Bershadsky, and G. V. Shivashankar, Proc. Natl. Acad. Sci. U. S. A. 112, E2595 (2015).
${ }^{19}$ S. M. Lim, J. P. Trzeciakowski, H. Sreenivasappa, L. J. Dangott, and A. Trache, Integr. Biol. 4, 615 (2012).

${ }^{20}$ O. Chaudhuri, S. H. Parekh, W. A. Lam, and D. A. Fletcher, Nat. Methods 6, 383 (2009).

${ }^{21}$ M. Caldarola, C. von Bilderling, M. E. Masip, L. I. Pietrasanta, and A. V. Bragas, in Proceedings of Frontiers in Optics 2012/Laser Science XXVIII (Optical Society of America, 2012).

${ }^{22}$ N. Q. Balaban, U. S. Schwarz, D. Riveline, P. Goichberg, G. Tzur, I. Sabanay, D. Mahalu, S. Safran, A. Bershadsky, L. Addadi, and B. Geiger, Nat. Cell Biol. 3, 466 (2001).

${ }^{23}$ B. D. Matthews, D. R. Overby, F. J. Alenghat, J. Karavitis, Y. Numaguchi, P. G. Allen, and D. E. Ingber, Biochem. Biophys. Res. Commun. 313, 758 (2004).

${ }^{24}$ D. Riveline, E. Zamir, N. Q. Balaban, U. S. Schwarz, T. Ishizaki, S. Narumiya, Z. Kam, B. Geiger, and A. D. Bershadsky, J. Cell Biol. 153, 1175 (2001).

${ }^{25}$ M. Tamada, M. P. Sheetz, and Y. Sawada, Dev. Cell 7, 709 (2004).

${ }^{26}$ N. Wang, J. P. Butler, and D. E. Ingber, Science 260, 1124 (1993).

${ }^{27}$ E. Zamir, M. Katz, Y. Posen, N. Erez, K. M. Yamada, B. Z. Katz, S. Lin, D. C. Lin, A. Bershadsky, Z. Kam, and B. Geiger, Nat. Cell Biol. 2, 191 (2000).

${ }^{28}$ J. L. Hutter and B. J. Bechhoefer, Rev. Sci. Instrum. 64, 1868 (1993).

${ }^{29}$ R. K. Ball, R. R. Friis, C. A. Schoenenberger, W. Doppler, and B. Groner, EMBO J. 7, 2089 (1988).

${ }^{30}$ C. Lawson and D. D. Schlaepfer, Cell Adhes. Migr. 6, 302 (2012).

${ }^{31}$ J. E. Hoffmann, Y. Fermin, R. L. Stricker, K. Ickstadt, and E. Zamir, eLife 3, e02257 (2014).

${ }^{32}$ C. T. Mierke, Phys. Biol. 10, 065005 (2013).

${ }^{33}$ M. D. Schaller, Oncogene 20, 6459 (2001).

${ }^{34}$ C. M. Laukaitis, D. J. Webb, K. Donais, and A. F. Horwitz, J. Cell Biol. 153 1427 (2001).

${ }^{35}$ G. von Wichert, B. Haimovich, G. S. Feng, and M. P. Sheetz, EMBO J. 22, 5023 (2003).

${ }^{36}$ R. Zaidel-Bar, C. Ballestrem, Z. Kam, and B. Geiger, J. Cell Sci. 116, 4605 (2003).

${ }^{37}$ S. E. Winograd-Katz, R. Fassler, B. Geiger, and K. R. Legate, Nat. Rev. Mol. Cell Biol. 15, 273 (2014).

${ }^{38}$ C. H. Yu, J. B. Law, M. Suryana, H. Y. Low, and M. P. Sheetz, Proc. Natl. Acad. Sci. U. S. A. 108, 20585 (2011).

${ }^{39}$ Z. Sun, S. Huang, Z. Li, and G. A. Meininger, Front. Physiol. 3, 472 (2012).

${ }^{40}$ B. D. Hoffman, C. Grashoff, and M. A. Schwartz, Nature 475, 316 (2011). 